TURKU-FL-P19-95

SISSA/AP/95/112

hep-ph/9509252

\title{
ON THE NONEQUILIBRIUM EFFECTIVE POTENTIAL
}

\author{
ANTONIO RIOTTO ${ }^{12}$ and IIRO VILJA目2) \\ 1) International School for Advanced Studies, SISSA-ISAS, \\ Strada Costiera 11, I-34014, Miramare, Trieste, Italy \\ and \\ Istituto Nazionale di Fisica Nucleare, Sezione di Padova, 35100 Padova, Italy \\ 2) Department of Physics, University of Turku, FIN-20500 Turku, Finland
}

\begin{abstract}
Nonequilibrium phenomena of the phase transitions are studied. It is shown that due to finite relaxation time of the particle distributions, the use of scalar background dependent distribution functions is inconsistent. This observation may change the picture of rapid processes during the electroweak phase transition, like subcritical bubble formation and propagation of bubble walls.
\end{abstract}

\footnotetext{
*riotto@tsmi19.sissa.it. Address after November 95: Theoretical Astrophysics Group, NASA/Fermilab, Batavia, IL60510, USA.

†vilja@utu.fi.
} 
The possibility that the Universe went through a series of phase transitions during its expansion and cooling down from temperatures close to the Planck scale has been intensively investigated in the past [1]. It is a common hope that many of the current questions of cosmology can be answered by studying the nontrivial dynamics of the approach to equilibrium in complex systems. Nevertheless, despite their immense relevance, only very recently more effort has been made to understand nonequilibrium phenomena occured in the early Universe.

Thermalization, reheating and relaxation are nonequilibrium phenomena that play crucial role in the completion of the inflationary era, which is thought to solve the horizon and the homogeneity problems [2] of modern cosmology. Recent investigations on the non-linear quantum dynamics of scalar fields have implications for the reheating and reveal that particle production appears to be significantly different from linear estimates due to the time evolution of the inflation field [3]. The quantum non-linear effects lead to an extremely effective dissipational dynamics and particle production even in the simplest self-interacting theory where the single particle decay is kinematically forbidden. Also they might help in alleviating the so-called Polony 四 problem concerning flat directions for some of the moduli fields in string theories.

At the electroweak scale, the focus has been in generation of the baryon asymmetry during a first-order phase transition where the $S U(2)_{L} \otimes U(1)_{Y}$ symmetry is broken down to $U(1)_{e m}$ [5]. It is currently believed that nonequilibrium conditions are a crucial ingredient for electroweak baryogenesis, even though there are certain questions related to the reliability of the perturbative expansion for weak transitions [6]. Moreover, the mechanism of the weak first-order transitions [0] is still an open question. Consider models with double-well potentials where the system starts localized on one minimum. For sufficiently weak transitions subcritical bubbles of the other phase could be thermally nucleated, giving rise to e.g. an effective phase mixing between the two available phases before cooling down to the tunneling temperature at which critical bubbles are expected to be nucleated. This may have dramatic consequences for any electroweak baryogenesis mechanism.

So far, however, most done to study the nonequilibrium aspects of phase transitions, e.g. the influence of subcritical bubbles on a first-order electroweak phase transition, is 
strictly related to the equilibrium finite temperature field theory [8] and effective potential which, by its very definition, is only adequate to describe equilibrium situations. Two equivalent methods are usually applied: the imaginary-time formalism (in Euclidean time) or the real-time formalism with equilibrium distribution functions. They are used to obtain the thermal part of the equilibrium effective potential by integrating out all the fermionic and bosonic degrees of freedom of the theory with $\phi_{c}$-dependent masses.

In the equilibrium case the finite temperature part of the one-loop effective potential reads [9] (besides an additive $\phi_{c}$-independent constant)

$$
V_{\mathrm{eq}}\left(T, \phi_{c}\right)=\sum_{i} \int \frac{d^{3} k}{(2 \pi)^{3}} \int_{\infty}^{\omega_{\mathbf{k}}\left(m_{i}\right)} d \omega f_{\mathrm{eq}}^{i}(\omega),
$$

where $\omega_{\mathbf{k}}\left(m_{i}\right)=\sqrt{|\mathbf{k}|^{2}+m_{i}^{2}\left(\phi_{c}\right)}, m_{i}\left(\phi_{c}\right)$ is the eigenstate of the mass matrix corresponding to the $i$-th degrees of freedom in a $\phi_{c}$-dependent background. Note, that $f_{\text {eq }}^{i}$ is the usual equilibrium Fermi-Dirac or Bose-Einstein distribution function. The effective potential $V_{\text {eq }}\left(T, \phi_{c}\right)$ represents the equilibrium free energy density as a function of the classical order parameter $\phi_{c}$ and can be used to determine the nature of the phase transition and static quantities like the critical temperature, but it is not the appropriate tool for the description of real-time processes, which are crucial to understand the mechanism by which the system approaches the equilibrium [10].

The aim of the present Letter is to point out that the use of the equilibrium finite temperature effective potential becomes completely unreliable when the thermalization time $\tau_{\text {th }}$ of the degrees of freedom getting a $\phi_{c}$-dependent mass in the thermal bath is larger than the typical time scale $\left|\phi_{c} / \dot{\phi}_{c}\right|$, I.e. when the macroscopic order parameter $\phi_{c}$ does not vary slowly in comparison with $\tau_{\text {th }}$. More generally, we can state that the equilibrium effective potential is not trustable at times $t$ smaller than the thermalization time $\tau_{\text {th }}$ in a $\phi_{c^{-}}$background changing in space and time at a generic instant $t=0$.

To avoid any confusion, we observe here that what we call thermalization time should be understood as the time needed for the degrees of freedom with a $\phi_{c}$-dependent mass to feel the change of the background $\phi_{c}$ and to relax their distribution functions to their equilibrium values $f_{\text {eq }}^{i}$ with the appropriate mass $m_{i}\left(\phi_{c}\right)$ [11]. Thus, for instance, the thermalization time for fermions is dictated by the Yukawa interactions with the scalar 
field $\phi$ and not by the strong interactions with gluons in the thermal bath, which are much faster.

In this paper we propose a nonequilibrium approach to describe the properties of the system in the specific limits depicted above and apply it to the Standard Model (SM) effective potential. We will also briefly discuss some physical situations in which our simple, but crucial, statement might have interesting consequences.

Let us first briefly clarify with a simple example our observation. Suppose a universe filled only with a real scalar field $\phi$ and a fermion field $\psi$ whose Lagrangian density reads

$$
\mathcal{L}=\frac{1}{2}\left(\partial_{\mu} \phi\right)^{2}+i \bar{\psi} \not \partial \psi-V(\phi)-g \phi \bar{\psi} \psi .
$$

The zero temperature tree level potential $V(\phi)$ is given by

$$
V(\phi)=-\frac{1}{2} \mu^{2} \phi^{2}+\frac{1}{4} \lambda \phi^{4} .
$$

At high temperatures the effective potential for the classical field $\langle\phi\rangle=\phi_{c}$ has the form

$$
V\left(T, \phi_{c}\right)=\frac{1}{2} m^{2}(T) \phi_{c}^{2}+\frac{1}{4} \lambda(T) \phi_{c}^{4},
$$

where

$$
m^{2}(T)=-\mu^{2}+\left(\frac{\lambda}{4}+\frac{g^{2}}{6}\right) T^{2},
$$

is the plasma mass for the $\phi_{c}$-field whereas $\lambda(T)$ receives only logarithmic one-loop corrections. Above the critical temperature $T_{c}$, the free energy of the system is minimized by $\phi_{c}=0$ and the Fermi-Dirac and Bose-Einstein distribution functions for the fermionic and bosonic degrees of freedom of the heat bath are the equilibrium ones

$$
\begin{aligned}
& f_{\psi}^{\mathrm{eq}}\left(\mathbf{k}, \phi_{c}=0\right)=\left(1+\mathrm{e}^{\beta|\mathbf{k}|}\right)^{-1} \\
& f_{\phi}^{\mathrm{eq}}\left(\mathbf{k}, \phi_{c}=0\right)=\left(\mathrm{e}^{\beta \sqrt{|\mathbf{k}|^{2}+m^{2}(T)}}-1\right)^{-1} .
\end{aligned}
$$

Let us now imagine that at a generic time $t=0$ in a certain region of space the vacuum expectation value becomes different from zero, $\phi_{c}=\phi_{c}(\mathbf{x}, t)$. This can happen because in that region the system suffers a thermal fluctuation or simply because the temperature of the thermal bath has cooled down to the critical temperature $T_{c}, \phi_{c}=0$ 
becomes an unstable point and $\phi_{c}$ starts to roll down. The equation of motion for $\phi_{c}(\mathbf{x}, t)$ reads

$$
\square \phi_{c}(\mathbf{x}, t)+\frac{\partial V\left[\phi_{c}, f_{\psi}, f_{\phi}\right]}{\partial \phi_{c}}=0,
$$

where $V\left(\phi_{c}\right)$ depends upon the distribution functions $f_{\psi}$ and $f_{\phi}$ through the one-loop corrections. The question is now whether we can use, for instance, the equilibrium distribution function $f_{\psi}^{\mathrm{eq}}\left(\phi_{c}\right)$ with a $\phi_{c}$-dependent mass $m_{\psi}\left(\phi_{c}\right)=g \phi_{c}$ to calculate the contribution of the fermionic degrees of freedom to the one-loop effective potential.

To answer the question one should solve the the Boltzmann equation

$$
\partial_{t} f_{\psi}+\dot{\mathbf{x}} \cdot \nabla f_{\psi}+\dot{\mathbf{k}} \cdot \partial_{\mathbf{k}} f_{\psi}=\mathcal{C}\left[f_{\psi}\right]
$$

where $\mathcal{C}\left[f_{\psi}\right]$ is the collision operator of the fermions. We define $\tau_{\text {th }}$ is the thermalization time for the fermionic degrees of freedom, i.e. the time needed for the fermions of the thermal bath to response to the change in the background $\phi_{c}$. By dividing for small times $0<\delta t<\tau_{\text {th }}$ the distribution function into two parts $f_{\psi}=f_{\psi}^{\mathrm{eq}}\left(\phi_{c}=0\right)+\delta f_{\psi}$ the lowest order correction $\delta f_{\psi}$ is determined by the collision operator with the distributions replaced with $f_{\psi}^{\mathrm{eq}}\left(\phi_{c}=0\right)$. The collision operator can be written in the form

$$
\mathcal{C}\left[f_{\psi}\right]=\int \frac{d^{3} q}{(2 \pi)^{3} E_{f}(q)} \frac{d^{3} k}{(2 \pi)^{3} E_{b}(k)}|\mathcal{M}|^{2} f_{\psi}^{\mathrm{eq}}(\mathbf{p}) f_{\psi}^{\mathrm{eq}}(\mathbf{q}) f_{\phi}^{\mathrm{eq}}(\mathbf{k})\left[e^{\beta \sqrt{|\mathbf{k}|^{2}+m^{2}}}-e^{\beta(|\mathbf{p}|+|\mathbf{q}|)}\right] .
$$

The dispersion relations are determined by rapid forward scatterings and thus the energy conservation relation is now given by $\sqrt{|\mathbf{k}|^{2}+m^{2}+3 \lambda \phi^{2}}=\sqrt{|\mathbf{p}|^{2}+g^{2} \phi^{2}}+\sqrt{|\mathbf{q}|^{2}+g^{2} \phi^{2}}$. Applying it, it is straightforward to convince oneself that the square bracket term in the collision operator given by (9) gives a contribution of the order $g^{2}$ or $\lambda$. Therefore, because also the matrix element is of the order $g^{2}$, one obtains the result that

$$
\frac{f_{\psi}}{f_{\psi}^{\mathrm{eq}}\left(\phi_{c}=0\right)}=1+\mathcal{O}\left(g^{4}\right)+\mathcal{O}\left(\lambda g^{2}\right) \text {. }
$$

On the contrary, expanding the $\phi_{c}$-dependent equilibrium distribution

$$
f_{\psi}^{\mathrm{eq}}\left(\phi_{c}\right)=\left(1+\mathrm{e}^{\beta \sqrt{|\mathbf{k}|^{2}+g^{2} \phi_{c}^{2}}}\right)^{-1}
$$

around $\phi_{c}=0$, one should get 


$$
\frac{f_{\psi}^{\mathrm{eq}}}{f_{\psi}^{\mathrm{eq}}\left(\phi_{c}=0\right)}=1+\mathcal{O}\left(g^{2}\right) .
$$

It is therefore clear that for times smaller than $\tau_{\text {th }}$, the distribution function $f_{\psi}$ is far from being equal to $f_{\psi}^{\mathrm{eq}}\left(\phi_{c}\right)$ and, as a consequence, the use of the equilibrium one-loop effective potential is not appropriate to describe the dynamics of the system.

This example contains the simple, but essential feature that thermalization takes time and, therefore, the degrees of freedom of the thermal bath can not always follow the change of the background $\phi_{c}$. Indeed, thermalization requires real scattering processes and therefore is usually fairly slow. Forward scatterings, instead, do not change the distribution functions of particles traversing a gas of quanta, but modify their free dispersion relations. This remains true also in the case of a nonequilibrium system. Forward scattering manifests itself, for example, as ensemble and scalar background corrections to the particle masses. Since the forward scattering rate is usually larger than the nonforward rate, non-equilibrium ensemble and scalar background corrections are present even for times smaller than the thermalization time. Afterwards, non-forward reaction rates become active and the system thermalizes.

Technically, the thermalization rate $\gamma_{\mathrm{th}}=\tau_{\mathrm{th}}^{-1}$ is related to the imaginary part of the two-point function via $\gamma_{\text {th }}=\operatorname{Im} \Gamma^{(2)}(\omega, \mathbf{k}) / \omega$. Here $\omega=\omega(\mathbf{k})$ is the solution to the dispersion relations where the scalar dependent mass is involved.

To decide how fast each particle species do thermalize, one should calculate the imaginary part of the two point function for each particle species separately. It is physically, however, clear that the rates are essentially the same than their contributions to the Higgs thermalization rate. Regardless what the actual rates are, the concept of non-equilibrium at short times remains. In the Standard Model, at one loop, the imaginary part of the Higgs propagator receives contributions from decay and absorption (emission) processes. Absorption (emission) is always proportional to the difference between the distribution functions of the two external particles in the final and initial state [12].

At high temperature limit, the thermalization rate of leptons with the Higgs background are negligible because of their small Yukawa couplings. Also for the quarks the thermalization rates with the Higgs background are small due to small Yukawa couplings apart the top quark which has the rate of the order $\gamma_{t} \simeq 10^{-2} T$ or smaller. At two 
loops kinematical constraints no longer exist. The leading, purely bosonic, contribution has been estimated in ref. [12] from the gauge boson loops and the thermalization rate for the gauge bosons with the Higgs background turns out to be at most of order of $\gamma_{\mathrm{gb}} \simeq 10^{-2} \mathrm{~T}$, whereas the thermalization rate of the scalar degrees of freedom receives a further suppression $\mathcal{O}\left(\lambda^{2} / g_{2}^{2}\right)$ with respect to $\gamma_{\mathrm{gb}}, \lambda$ being the quartic self-coupling of the Higgs scalar potential and $g_{2}$ the $S U(2)_{L}$ gauge coupling. Thus, because of both kinematical and loop suppression factors, the thermalization rate of fermions, gauge and scalar bosons with the Higgs background turns out to be fairly small.

Let us now envisage the situation in which the $\phi_{c}$-background, permeating the thermal bath formed by SM degrees of freedom, changes from $\phi_{c}=0$ to a non-vanishing value. From general point of view the initial field value could be arbitrary with initial distribution determined by that. In practise the most of the interesting cases have initially $\phi=0$ and the applications we consider use that initial value. A realistic example for such a situation could be the formation of a subcritical bubble via thermal fluctuations above the transition temperature $T_{f}$ or the passage at a given point of the wall of an expanding critical bubble nucleated at $T_{f}$ in a first-order electroweak phase transition.

As said above, we can not use the imaginary-time formalism to compute the effective Higgs potential for times smaller than $\sim \gamma_{\mathrm{th}}^{-1}$ since there is no relation between the density matrix of the system and the time evolution operator which is of essential importance in the equilibrium case. There is, however, the real-time formalism of Thermo Field Dynamics which suites our purposes [13] and is characterized by doubling the degrees of freedom of the heat reservoir. It is straightforward to find the (11)-component of the scalar and fermion propagators for the fields

$$
\begin{aligned}
& D_{\mathrm{s}}(k)=\frac{i}{k^{2}-m^{2}\left(\phi_{c}\right)+i \varepsilon}+2 \pi \delta\left[k^{2}-m^{2}\left(\phi_{c}\right)\right] f_{\mathrm{b}}(k), \\
& D_{\mathrm{f}}(k)=\frac{i\left[k k+m\left(\phi_{c}\right)\right]}{k^{2}-m^{2}\left(\phi_{c}\right)+i \varepsilon}-2 \pi \delta\left[k^{2}-m^{2}\left(\phi_{c}\right)\right]\left[\not k+m\left(\phi_{c}\right)\right] f_{\mathrm{f}}(k),
\end{aligned}
$$

and analogous formulae for the gauge boson propagators. Note, that here $f_{\mathrm{b}, \mathrm{f}}(k)$ are arbitrary distribution functions restricted by requiring the number expectation value to be positive. 
The general expression for the thermal part of the effective potential can be calculated by usual way (e.g. by the tadpole method) but the calculation leads now to a different expression for the potential than Eq. (1) (with $f_{\text {eq }}^{i}$ replaced by the generic expression $\left.f_{\mathrm{b}(\mathrm{f})}\right)$. The derivation of formula (I) uses explicitly the concept of thermal equilibrium [9] or, as in the case of tadpole method in real time formalism, it is assumed that the distribution is a function of energy, i.e. it is $\phi_{c}$ dependent. Now, for times smaller than $\gamma_{\mathrm{th}}^{-1}, f_{\mathrm{b}, \mathrm{f}}$ are well approximated by

$$
f_{\mathrm{b}, \mathrm{f}}^{\mathrm{eq}}=\left(\mathrm{e}^{\sqrt{|\mathbf{k}|^{2}+m^{2}\left(T, \phi_{c}=0\right)} / T} \mp 1\right)^{-1}
$$

where $m\left(T, \phi_{c}\right)$ is the plasma mass at finite temperature for each degree of freedom.

The choice given in Eq. (14) for the distribution functions needs some justification: at $t<\gamma_{\text {th }}^{-1}$ particles have not had time enough to have real scatterings, and thus the distributions have not yet had time enough to feel the change of the background $\phi_{c}$. So they remains the same as they were when the background mass was $\phi_{c}=0$. Nevertheless, since forward scattering are much faster than non-forward reaction rates, the free dispersion relation of particles get modified and their masses receive plasma corrections from the ensemble. Furthermore, since quanta interactions are rather fast, e.g. mediated by strong force, the use of the equilibrium distribution function is well motivated. We refer the reader to ref. [14] for more details.

Calculating the effective potential, we find

$$
V\left(T, \phi_{c}\right)=\frac{1}{2 \pi^{2}} \operatorname{Tr} \int_{0}^{\infty} d|\mathbf{k}||\mathbf{k}|^{2} \sqrt{|\mathbf{k}|^{2}+m^{2}\left(T, \phi_{c}\right)}\left(\mathrm{e}^{\sqrt{|\mathbf{k}|^{2}+m^{2}\left(T, \phi_{c}=0\right)} / T} \mp 1\right)^{-1},
$$

where the trace is taken over all degrees of freedom and $m^{2}\left(T, \phi_{c}\right)$ is a general mass matrix. Same techniques as used in the calculation of equilibrium potential [9] can be applied here resulting a general formula in which the leading terms are included

$$
V\left(T, \phi_{c}\right)=\frac{T^{4}}{2 \pi^{2}}\left[\operatorname{Tr} I_{\mathrm{b}}\left(\frac{m_{\mathrm{b}}\left(T, \phi_{c}\right)}{T}, \frac{m_{\mathrm{b}}\left(T, \phi_{c}=0\right)}{T}\right)+\operatorname{Tr} I_{\mathrm{f}}\left(\frac{m_{\mathrm{f}}\left(T, \phi_{c}\right)}{T}, \frac{m_{\mathrm{f}}\left(T, \phi_{c}=0\right)}{T}\right)\right] .
$$

The bosonic (fermionic) functions $I_{\mathrm{b}(\mathrm{f})}$ are given by

$I_{\mathrm{b}}(x, y)=\frac{1}{2}\left[\frac{1}{6} \pi^{2}-y+\frac{1}{4} y^{2}\right] x^{2}-\frac{1}{3} x^{3}+\frac{1}{16}\left[\frac{3}{4}-1-\gamma_{E}+\ln (4 \pi)\right] x^{4}-\frac{1}{32} x^{4} \ln x^{2}+\ldots$ 
and

$$
I_{\mathrm{f}}(x, y)=\left[\frac{1}{24} \pi^{2}-\frac{1}{8} y^{2}\right] x^{2}+\frac{1}{16}\left[\frac{1}{4}+\gamma_{E}-\ln (\pi)\right] x^{4}+\frac{1}{32} x^{4} \ln x^{2}+\ldots
$$

Here $\gamma_{E}$ is the Euler constant and $m_{\mathrm{b}(\mathrm{f})}$ is a general mass matrix of bosonic (fermionic) degrees of freedom. In the formulas above the dots stand for higher powers of $x$. It is worth to note that in the mass matrices we are able to use the temperature dependent masses: the resummation can be done similarly than in the equilibrium case [6]. This potential clearly differs from the usual equilibrium potential, whereas the zero-temperature part remains naturally unaltered. In particular, the next-to-leading boson contribution coming from the infrared region to the cubic term differs from the equilibrum one by a factor $2 / \pi$.

After adding up the two parts we have plotted the effective potential for the Standard Model in the particular case of $m_{H}=65 \mathrm{GeV}$, Fig. 1. The critical temperature $T_{c}$ defined as the temperature at which $V\left(T_{c}, 0\right)=V\left(T_{c}, \phi_{c}^{+}\right), \phi_{c}^{+}$being the non-vanishing minimum, is around $94.27 \mathrm{GeV}$, higher than the critical temperature for the equilibrium case (around $90 \mathrm{GeV}$ ) for the same choice of the Higgs mass, see [14] for a detail analysis.

Let us now briefly discuss two physical cases in which our nonequilibrium approach to the effective potential might qualitatively effect the conclusions commonly drawn employing the equilibrium effective potential.

As already mentioned, the role played by subcritical bubbles at the onset of a firstorder electroweak phase transition is still disputed [7]. It might be possible that the amplitude of thermal fluctuations be so large that the fraction occupied by the symmetric minimum in the neighborhood of the critical temperature $T_{c}$ becomes of order unity, thus preventing the formation of expanding critical bubbles and hindering any mechanism for electroweak baryogenesis.

To compute the average amplitude of thermal fluctuations it is commonly hypothesized that the free energy $\mathrm{F}$ of a subcritical bubble configuration $\phi_{\mathrm{sb}}$ receives contribution from the equilibrium effective potential. This gives rise to a Boltzmann weight $\sim \exp \left[-\mathrm{F}\left(\phi_{\mathrm{sb}}\right) / T\right]$ for each configuration. However, subcritical bubbles, being unstable objects, tend to shrink. It is rather conceivable that thermal fluctuations are dominated by large-size configurations whose lifetime can be roughly estimated to be $\tau_{\mathrm{sh}} \sim m_{H}^{-1}(T)$. 
Since $\tau_{\text {sh }} \ll \gamma_{\text {th }}^{-1}$ at the critical temperature, particles inside the subcritical bubble do not experience thermalization with the $\phi_{\mathrm{sb}}$-configuration. Consequently, the equilibrium effective potential is completely inadequate to determine the free energy of such configurations and must be taken over by the nonequilibrium one.

Our nonequilibrium approach might also have consequences for the determination of the velocity $v_{\mathrm{w}}$ and width $L_{\mathrm{w}}$ of critical bubble walls expanding in the thermal bath at the onset of a first-order electroweak phase transition. The determination of these parameters has received much attention [15] since the discovery of the possibility to generate the baryon asymmetry at the electroweak scale. Intuitively speaking, the velocity and the shape of the bubble wall depend upon two factors: the pressure difference $\Delta p$ at the two edges of the bubble wall (i.e. between the broken and the unbroken phase), which allows for the expansion, and the friction force due to the collisions of the plasma particles off the wall. In the previous treatments, the population density $f^{i}$ of each species $i$ has been splitted in the equilibrium one plus a small deviation, $f^{i}=f_{\mathrm{eq}}^{i}\left(\phi_{c}\right)+\delta f^{i}$. The pressure difference $\Delta p$ is then determined by $\Delta V_{\text {eq }}\left(\phi_{c}\right)$ and the friction force arises due to departure from the thermal equilibrium distribution. However, since thermalization between the degrees of freedom of the thermal bath and the bubble wall background takes a finite time, this picture is correct only if $\gamma_{\mathrm{th}}^{-1} \ll\left(L_{\mathrm{w}} / v_{\mathrm{w}}\right)$. If the opposite limit, equilibrium is not attained in the vicinity of the bubble wall in the broken phase and the nonequilibrium effective potential should play a role in estimating the pressure difference $\Delta p$ between the two phases.

We are currently investigating both issues [14].

\section{ACKNOWLEDGMENTS}

The authors thank the organizers of the TAN Workshop held in Vigsøe, August 1995, where part of this work was done. 


\section{REFERENCES}

[1] For a review see E. W. Kolb and M. S. Turner,. The Early Universe, (AddisonWesley, Redwood, CA, 1990).

[2] For a review see K. Olive, Phys. Rep. 190 (1990) 307.

[3] D. Boyanovsky, H.J. de Vega, Phys. Rev. D47 (1993) 2343; D. Boyanovsky, D.-S. Lee and A. Singh, Phys. Rev. D48 (1993) 800; D. Boyanovsky, H.J. de Vega and R. Holman, Phys. Rev. D49 (1994) 2769 and Proceedings of the Second Paris Cosmology Colloquium, Observatoire de Paris, June 1994, p. 125-215, H.J. de Vega and N. Sanchez Editors, World Scientific, 1995; L. Kofman, A.D. Linde and A.A. Starobinsky, Phys. Rev. Lett. 73 (1994) 3195; Y. Shtanov, J. Traschen and R. Brandenberger, Phys. Rev. D51 (1995) 5438; A. Dolgov and K. Freese, Phys. Rev. D51 (1995) 2693; D. Boyanovsky, H.J. de Vega, R. Holman, D.-S. Lee and A. Singh, Phys. Rev. D51 (1995) 4419; D. Boyanovsky, M. D’Attanasio, H.J. de Vega, R. Holman and D.-S. Lee and A. Singh, hep-ph/9505220; D. Boyanovsky, M. D'Attanasio, H.J. de Vega, R. Holman and D.-S. Lee, hep-ph/9507414.

[4] See, for instance, T. Banks et al., hep-ph/9501053 and references therein.

[5] A.G. Cohen, D.B. Kaplan, and A.E. Nelson, Annu. Rev. Nucl. Part. Sci. 43 (1993) 27; A. Dolgov, Phys. Rep. 222, (1992) 311.

[6] For a review see, M. Quirós, Field theories at finite temperature and Phase Transitions, Helvetica Physica Acta 67 (1994) 451 and references therein.

[7] M. Gleiser, E.W. Kolb and R. Watkins, Nucl. Phys. B364, 411 (1991); M. Gleiser and E.W. Kolb, Phys. Rev. Lett. 69, 1304 (1992); Phys. Rev. D48, 1560 (1993); M. Dine, R. Leigh, P. Huet, A. Linde and D. Linde, Phys. Rev. D46, 550 (1992); G. Anderson, Phys. Lett. B295, 32 (1992); M. Gleiser and R.O. Ramos, Phys. Lett. B300, 271 (1993); N. Tetradis, Z. Phys. C57, 331 (1993); M. Gleiser, Phys. Rev. Lett. 73 (1994) 3495; G. Gelmini and M. Gleiser, Nucl. Phys. B419, 459 (1994); J. Borril and M. Gleiser, Phys. Rev. D51 (1995) 4111; K. Enqvist and I. Vilja, Phys. Lett. B344 (1995) 98; J. Sirkka and I. Vilja, Phys. Lett. B354 (1995) 85; K. Enqvist, A. Riotto and I. Vilja, hep-ph 9505341, accepted for publication 
in Phys. Rev. D; F. Illuminati and A. Riotto, hep-ph 9506419, submitted to Nucl. Phys. B; L.M.A. Bettencourt, Phys. Lett. B356 (1995) 297; M. Gleiser, DARTHEP-95/03 preprint, talk given at the 3éme Colloque Cosmologie, Paris, June 7-9, 1995.

[8] For a thorough review see J. Kapusta, Finite Temperature Field Theory, Cambridge Univ. Press, 1989.

[9] L. Dolan and R. Jackiw, Phys. Rev. D9 (1974) 3320.

[10] A. Hosoya and M. Sakagami, Phys. Rev. D29 (1984) 2228; M. Morikawa, Phys. Rev. D33 (1986) 3607; M. Gleiser and R. Ramos, Phys. Rev. D50 (1994) 2441; B.L. Hu, J.P. Paz and Y. Zhang, in The Origin of Structure in the Universe, Ed. E. Gunzig and P. Nardone Kluwer Acad. Publ. 1993; D. Lee and D. Boyanovsky, Nucl. Phys. B406 (1993) 631; S. Habib, in Stochastic Processes in Astrophysics, Proc. Eighth Annual Workshop in Nonlinear Astronomy, 1993.

[11] H.A. Weldon, Phys. Rev. D28 (1983) 2007.

[12] P. Elmfors, K. Enqvist and I. Vilja, Nucl. Phys. B412 (1994) 459. Here the thermalization rate was estimated on-shell and for vanishing spatial momentum. This is, however, the interesting limit for practical purposes.

[13] H. Umezawa, H. Matsumoto and M. Tachiki, Thermo Field Dynamics and Condensed States, North Holland, 1982. See also, P. Elmfors, K. Enqvist and I. Vilja, Phys. Lett. B326 (1994) 37.

[14] A. Riotto and I. Vilja, in preparation.

[15] See, for distance, G.D. Moore and T. Prokopec, Phys. Rev. Lett. 75 (1995) 777; hep-ph 9506475 and references therein. 


\section{Figure Captions}

Fig. 1: The Standard Model nonequilibrium effective potential (in units of $\mathrm{GeV}$ ) for the particular choice $m_{H}=65 \mathrm{GeV}$. 


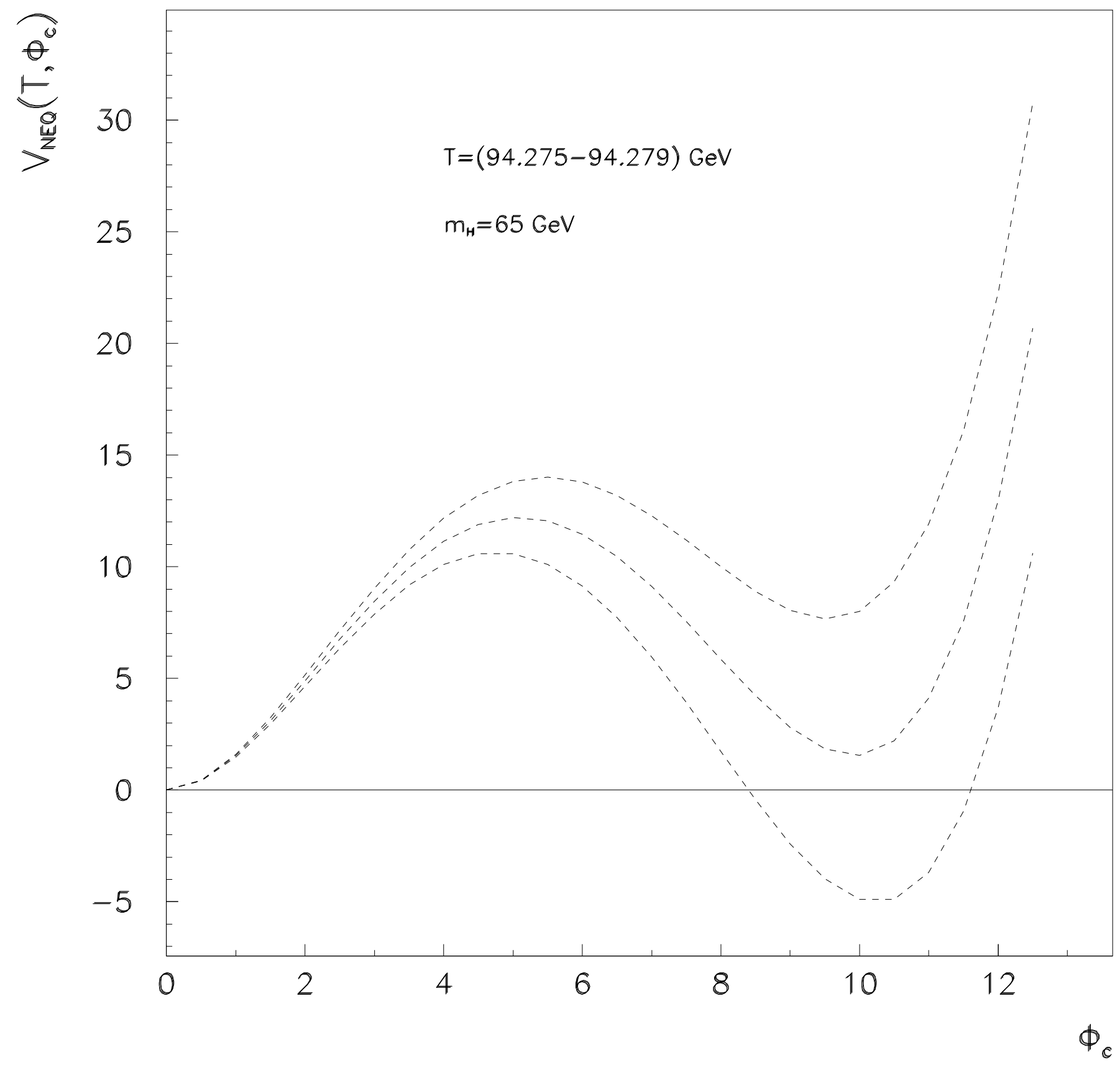

